OPEN ACCESS

Edited by:

Michal Masternak,

University of Central Florida,

United States

Reviewed by:

Alice Masini,

University of Bologna, Italy

Jessica M. Hoffman,

University of Alabama at Birmingham,

United States

Rita Ostan

University of Bologna, Italy

*Correspondence:

Jinwen Tian

tjssqr.2000@163.com

Yali Zhao

zhaoyl301@163.com

Yao Yao

yaoyao@nsd.pku.edu.cn

${ }^{\dagger}$ These authors share first authorship

Specialty section: This article was submitted to

Endocrinology of Aging,

a section of the journal

Frontiers in Endocrinology

Received: 11 October 2020 Accepted: 14 December 2020

Published: 03 February 2021

Citation:

Zhu Q, Fu S, Zhang Q, Tian J, Zhao Y and Yao Y (2021) Female Fertility Has a Negative Relationship With Longevity in Chinese Oldest-Old Population: A Cross-Sectional Study.

Front. Endocrinol. 11:616207. doi: 10.3389/fendo.2020.616207

\section{Female Fertility Has a Negative Relationship With Longevity in Chinese Oldest-Old Population: A Cross-Sectional Study}

\author{
Qiao Zhu ${ }^{1+}$, Shihui $\mathrm{Fu}^{2+}$, Qian Zhang ${ }^{3 \dagger}$, Jinwen Tian ${ }^{2 *}$, Yali Zhao ${ }^{1 *}$ and Yao Yao ${ }^{4,5 *}$ \\ ${ }^{1}$ Central Laboratory, Hainan Hospital of Chinese People's Liberation Army General Hospital, Sanya, China, ${ }^{2}$ Department of \\ Cardiology, Hainan Hospital of Chinese People's Liberation Army General Hospital, Sanya, China, ${ }^{3}$ Department of \\ Neurology, Hainan Hospital of Chinese People's Liberation Army General Hospital, Sanya, China, ${ }^{4}$ Center for Healthy Aging \\ and Development Studies, Raissun Institute for Advanced Studies, National School of Development, Peking University, \\ Beijing, China, ${ }^{5}$ Center for the Study of Aging and Human Development and Geriatrics Division, Medical School of Duke \\ University, Durham, NC, United States
}

Background: Despite research efforts in this field for more than a century, the relationship between female fertility and longevity is unclear. This study was designed to investigate this relationship in Chinese oldest-old population.

Methods: The China Hainan Centenarian Cohort Study was performed in 18 cities and counties of Hainan. A total of 1,226 females, including 758 centenarian women and 468 women aged 80-99 years, were enrolled in this study. Using a standardized protocol, inperson interviews and blood analyses were conducted by a well-trained research team through home visits.

Results: Centenarian women had significantly lower number of children (NOC) and higher initial childbearing age (ICA) and last childbearing age (LCA) than women aged 80-99 years $(p<0.05$ for all). Multivariate logistic regression analysis showed that NOC and testosterone (T) levels were positively associated with women aged 80-99 years, when centenarian women was considered as reference ( $p<0.05$ for all). ICA, LCA, and estradiol (E2) levels were negatively associated with women aged 80-99 years, when centenarian women was considered as reference $(p<0.05$ for all).

Conclusions: The centenarians had crucial characteristics of less and delayed childbearing, indicating a negative relationship between female fertility and longevity in Chinese oldest-old population. Serum E2 levels were positively associated and serum T levels were negatively associated with longevity. The less and late childbearing might be a significant factor of longevity, and successful aging might be promoted by reducing and delaying female childbearing.

Keywords: female fertility, oldest-old population, centenarian, longevity, negative relationship 


\section{INTRODUCTION}

Antagonistic pleiotropy and disposable soma theories have suggested that female fertility is inversely related to life span because there is a metabolic trade-off between reproduction and longevity $(1,2)$. Disposable soma theory has suggested that aging is a dynamic process with two aspects: random damage and repair of deoxyribonucleic acid in the process of life $(3,4)$. The human body is affected by a variety of external and internal damages; aging is accompanied by accumulated damage and continuous repair. When the repair cannot make up for the damage due to the energy shortage caused by growth and reproduction, the body will eventually die (5). Meanwhile, antagonistic pleiotropy theory has suggested that the gene has multiple functions, some of which are beneficial to growth and reproduction and some of which are not (6). Evolutionary selection of mutated genes helps people grow and reproduce when they are young, but these genes lead people to age with time because the main function has been achieved, i.e., passing genetic information on to the next generation (7). Previous studies have shown a negative relationship between female fertility and life span because of the high energy expenditure in female reproduction (8-13). In the Caerphilly study, female fertility has been found to be negatively correlated with postreproductive longevity $(14,15)$. However, other studies have found no significant relationship between female fertility and life span $(16,17)$. A contemporary study involving Norwegians have also highlighted a positive relationship between female fertility and post-reproductive longevity $(18,19)$. Despite research efforts in this field for more than a century, this relationship is unclear. Hence, there is a need for studies clarifying the relationship between female fertility and longevity.

Centenarians represent a prototype of successful aging, and centenarian women experience an appropriate pattern of fertility (20-23). It might be worth noting the characteristics of female fertility in the centenarians to identify ways to promote successful aging and human longevity. There is growing interest in female fertility in centenarians. To our knowledge, no study has explained the relationship between female fertility and longevity, especially in Chinese oldest-old population (24). Hainan is an area with the highest population density of centenarians in China. The China Hainan Centenarian Cohort Study (CHCCS), with a considerable sample size, provides a significant population-based sample of Chinese centenarians (25). This study was designed to investigate the relationship between female fertility and longevity in Chinese oldestold population.

\section{METHODS}

\section{Study Population}

As a population-based study, the CHCCS was carried out in 18 cities and counties of Hainan, China, from 2014 to 2017. Its cohort profile has been described previously (25). In brief, a total of 1,226 females, including 758 centenarian women and 468 women aged 80-99 years, were enrolled in this study. All participants were naturally aging and never took hormone drugs. This study was approved by the Ethics Committee of Hainan Hospital of Chinese People's Liberation Army General Hospital (Sanya, Hainan; Number: 301hn11201601). Written informed consent was obtained from each participant prior to the start of this study.

\section{Standard Procedures}

Using a standardized protocol, in-person interviews and blood analyses were conducted by a well-trained research team at Chinese People's Liberation Army General Hospital through home visits. This interdisciplinary research team included internists, geriatricians, cardiologists, endocrinologists, nephrologists, and nurses. Ethnicity was categorized as either ethnic Han (the predominant ethnicity in China) or others. Education was categorized as illiterate or literate, where illiterate indicated the inability to read and write. Fertility status and age were provided by the participants themselves or friends and relatives of the advanced age, and verified by the number and age of offspring and related population records and medical information. Number of children (NOC) referred to the number of offspring born alive. Initial childbearing age (ICA) referred to the age at which the woman gave birth to the first live offspring. Last childbearing age (LCA) referred to the age at which the woman gave birth to the last live offspring. Barthel Index (BI) was used to evaluate the activities of daily living (ADL); its validity and reliability in Chinese older individuals have been well established (26). BI consists of 10 items that measure a person's daily activities: grooming, feeding, dressing, bathing, toilet use, transfer from bed to chair, walking, stair climbing, bowel continence, and urinary continence. Each item of $\mathrm{BI}$ is rated on a scale with points assigned to each level of activity, and the total score ranges from 0 to 100 points with 5point increments. A high score indicates a high level of ADL. EuroQol 5 Dimensions (EQ-5D) was used to evaluate the qualities of daily living. EQ-5D covers five dimensions: mobility, self-care, usual activities, pain/discomfort, and anxiety/depression. The standardized index of EQ-5D is based on Chinese data, and validated in various populations with good reliability, with a high score indicating a high quality of daily living (27). Body mass index (BMI) was defined as the weight in kilograms divided by the square of the height in meters. Blood samples were routinely drawn by venipuncture and stored in serum separator tube at $4^{\circ} \mathrm{C}$ to obtain the serum and transported to the central laboratory in our Biochemistry Department within 4 h. Electrochemiluminescence (Cobas E602; Roche Products Ltd, Basel, Switzerland) was used to determine serum levels of estradiol (E2), testosterone (T), follicle-stimulating hormone (FSH), and luteinizing hormone (LH). Serum levels of total cholesterol (TC), triglyceride (TG), high-density lipoprotein cholesterol (HDL-C), low-density lipoprotein cholesterol (LDLC), and blood glucose (Glu) were also determined by qualified technicians who were blinded to clinical data. 


\section{Statistical Analyses}

EpiData 3.0 software was used to store all the data on a designated computer. Based on $\alpha=0.05$ and $\beta=0.95$, the sample size was calculated to be more than 143 individuals for each group. Continuous variables with normal distribution were expressed as mean and standard deviation and compared using student's t test. Categorical variables were expressed as count and percentage and compared using Chi-square test or Fisher's exact test. Multivariate logistic regression analysis was carried out to explore fertility status and sex hormones associated with women aged 80-99 years, when centenarian women was considered as reference, after adjusting for ethnicity, education, smoking, drinking, meal times, BI, EQ-5D, BMI, systolic blood pressure (SBP), diastolic blood pressure (DBP), TC, TG, HDL-C, LDL-C, and Glu levels. Statistical analyses were performed with Statistical Package for Social Science (SPSS) software (IBM, NY, USA) and Power Analysis and Sample Size (PASS) software (NCSS, UT, USA). Two-tailed $\mathrm{P}<0.05$ indicated statistical significance.

\section{RESULTS}

The median age of the study population was $98.28 \pm 9.17$ years (range: 80-116). As shown in Table 1, centenarian women had significantly lower NOC and higher ICA and LCA than women aged 80-99 years ( $p<0.05$ for all). Serum E2 levels were significantly higher and serum $\mathrm{T}$ levels were significantly lower in the centenarian women than in the women aged 80-99 years
( $\mathrm{P}<0.05$ for all). Proportions of illiterate status and smoking were significantly higher, meal times were significantly more, age, SBP, and Glu levels were significantly higher, and BMI, BI, EQ-5D, DBP TC, TG, and LDL-C levels were significantly lower in the centenarian women than in the women aged 80-99 years $(\mathrm{P}<0.05$ for all). FSH, LH, and HDL-C levels, and proportions of ethnic Han and drinking showed no significant difference between centenarian women and women aged 80-99 years (Table 1; P > 0.05 for all).

Multivariate logistic regression analysis showed that NOC and $\mathrm{T}$ levels were positively associated with women aged 80-99 years, when centenarian women was considered as reference, suggesting that lower NOC and T levels were significantly associated with longevity, after adjusting for ethnicity, education, smoking, drinking, meal times, BI, EQ-5D, BMI, SBP, DBP, TC, TG, HDL-C, LDL-C, and Glu levels (Table 2; $\mathrm{p}<0.05$ for all). ICA, LCA, and E2 levels were negatively associated with women aged 80-99 years, when centenarian women was considered as reference, suggesting that higher ICA, LCA, and E2 levels were significantly associated with longevity, after adjusting for ethnicity, education, smoking, drinking, meal times, BI, EQ-5D, BMI, SBP, DBP, TC, TG, HDL-C, LDL-C, and Glu levels (Table 2; $\mathrm{p}<0.05$ for all).

\section{DISCUSSION}

Aging is a gradual process whereby the human body loses its ability to physiological repair itself, fertility declines, and the

TABLE 1 | Characteristics of oldest-old population aged 80-99 years and $\geq 100$ years.

\begin{tabular}{|c|c|c|c|c|}
\hline Characteristics & Total $(\mathrm{N}=1226)$ & $80-99$ years $(N=468)$ & $\geq 100$ years $(N=758)$ & $P$ value \\
\hline Age, years & $98.28 \pm 9.17$ & $85.55 \pm 4.52$ & $102.91 \pm 2.87$ & $<0.001$ \\
\hline Ethnic Han, n (\%) & 1,086 (88.6) & $414(88.5)$ & $672(88.7)$ & 0.927 \\
\hline Illiterate status, n (\%) & $1,164(94.9)$ & 432 (92.3) & 732 (96.6) & 0.001 \\
\hline Smoking, n (\%) & $65(5.3)$ & $13(2.8)$ & $52(6.9)$ & 0.002 \\
\hline Drinking, n (\%) & $183(14.9)$ & $80(17.1)$ & $103(13.6)$ & 0.099 \\
\hline Meal times & $2.99 \pm 0.55$ & $2.96 \pm 0.54$ & $3.03 \pm 0.57$ & 0.034 \\
\hline $\mathrm{Bl}$ & $81.21 \pm 23.33$ & $93.22 \pm 13.75$ & $73.79 \pm 24.90$ & $<0.001$ \\
\hline EQ-5D & $0.74 \pm 0.23$ & $0.87 \pm 0.17$ & $0.67 \pm 0.23$ & $<0.001$ \\
\hline NOC, n (\%) & $4.6 \pm 2.1$ & $5.2 \pm 2.0$ & $4.3 \pm 2.2$ & $<0.001$ \\
\hline ICA, years & $27.6 \pm 6.7$ & $25.5 \pm 4.5$ & $28.9 \pm 7.4$ & $<0.001$ \\
\hline LCA, years & $41.8 \pm 7.4$ & $39.8 \pm 5.5$ & $43.0 \pm 8.1$ & $<0.001$ \\
\hline $\mathrm{BMl}, \mathrm{kg} / \mathrm{m}^{2}$ & $19.21 \pm 3.77$ & $20.68 \pm 3.88$ & $18.30 \pm 3.39$ & $<0.001$ \\
\hline $\mathrm{SBP}, \mathrm{mmHg}$ & $153.30 \pm 24.31$ & $151.47 \pm 22.89$ & $154.44 \pm 25.09$ & 0.034 \\
\hline $\mathrm{DBP}, \mathrm{mmHg}$ & $78.22 \pm 13.76$ & $80.97 \pm 12.79$ & $76.51 \pm 14.39$ & $<0.001$ \\
\hline $\mathrm{E} 2, \mathrm{pmol} / \mathrm{L}$ & $35.50 \pm 25.99$ & $25.50 \pm 13.39$ & $41.67 \pm 29.71$ & $<0.001$ \\
\hline $\mathrm{T}, \mathrm{nmol} / \mathrm{L}$ & $0.86 \pm 1.63$ & $0.90 \pm 1.94$ & $0.67 \pm 1.35$ & 0.048 \\
\hline FSH, IU/L & $83.27 \pm 28.90$ & $84.32 \pm 37.30$ & $82.60 \pm 29.83$ & 0.210 \\
\hline $\mathrm{LH}, \mathrm{mlU} / \mathrm{ml}$ & $37.84 \pm 17.35$ & $37.13 \pm 13.53$ & $38.27 \pm 14.82$ & 0.184 \\
\hline TC, mmol/L & $4.94 \pm 1.09$ & $5.23 \pm 1.13$ & $4.76 \pm 1.03$ & $<0.001$ \\
\hline TG, mmol/L & $1.27 \pm 0.74$ & $1.35 \pm 0.77$ & $1.22 \pm 0.71$ & 0.002 \\
\hline HDL-C, mmol/L & $1.45 \pm 0.41$ & $1.45 \pm 0.41$ & $1.46 \pm 0.40$ & 0.869 \\
\hline LDL-C, mmol/L & $2.97 \pm 0.88$ & $3.15 \pm 0.97$ & $2.85 \pm 0.81$ & $<0.001$ \\
\hline Glu, mmol/L & $5.00 \pm 1.72$ & $4.85 \pm 1.90$ & $5.09 \pm 1.59$ & 0.016 \\
\hline
\end{tabular}

Data were expressed as mean \pm standard deviation or count (percentage).

BI, Barthel Index; EQ-5D, EuroQol 5 Dimensions; NOC, number of children; ICA, initial childbearing age; LCA, last childbearing age; BMI, body mass index; SBP, systolic blood pressure; $D B P$, diastolic blood pressure; E2, estradiol; T, testosterone; FSH, follicle-stimulating hormone; LH, luteinizing hormone; TC, total cholesterol; TG, triglyceride; HDL-C, high-density lipoprotein cholesterol; LDL-C, low-density lipoprotein cholesterol; Glu, blood glucose. 
TABLE 2 | Fertility status and sex hormone associated with women aged 80-99 years in logistic regression analysis with centenarian women as reference.

\begin{tabular}{|c|c|c|c|c|}
\hline Variables & B & OR & $95 \% \mathrm{Cl}$ & $P$ value \\
\hline NOC, n (\%) & 0.288 & 1.33 & $1.17-1.53$ & $<0.001$ \\
\hline ICA, years & -0.062 & 0.94 & $0.91-0.97$ & $<0.001$ \\
\hline LCA, years & -0.121 & 0.89 & $0.86-0.91$ & $<0.001$ \\
\hline $\mathrm{E} 2, \mathrm{pmol} / \mathrm{L}$ & -0.045 & 0.96 & $0.94-0.97$ & $<0.001$ \\
\hline $\mathrm{T}, \mathrm{nmol} / \mathrm{L}$ & 0.162 & 1.18 & $1.07-1.29$ & 0.001 \\
\hline FSH, IU/L & 0.005 & 1.01 & 0.99-1.01 & 0.287 \\
\hline $\mathrm{LH}, \mathrm{mlU} / \mathrm{ml}$ & 0.001 & 1.00 & $0.98-1.02$ & 0.902 \\
\hline
\end{tabular}

Fertility status and sex hormone associated with women aged 80-99 years in the multivariate logistic regression analysis, when centenarian women were considered as reference, after adjusting for ethnicity, education, smoking, drinking, meal times, BI, EQ-5D, BMI, SBP, DBP, TC, TG, HDL-C, LDL-C, and Glu levels.

$O R$, odds ratio; $C l$, confidential interval; NOC, number of children; ICA, initial childbearing age; LCA, last childbearing age; E2, estradiol; T, testosterone; FSH, follicle-stimulating hormone; LH, luteinizing hormone; BI, Barthel Index; EQ-5D, EuroQol 5 Dimensions; BMI, body mass index; SBP, systolic blood pressure; DBP, diastolic blood pressure; TC, total cholesterol;

TG, triglyceride; HDL-C, high-density lipoprotein cholesterol; LDL-C, low-density lipoprotein cholesterol; Glu, blood glucose.

probability of death increases. The evolutionary theories of aging have suggested that the rate of intrinsic aging is associated with a decline in fertility and survival (28). According to disposable soma theory, there is an evolutionary trade-off between fertility and longevity due to the energy requirements for both repair and fertility. However, due to a lack of convincing evidence, the relationships between fertility and longevity have attracted researchers' attention for more than a century. Some studies have shown a negative correlation between NOC and life span because of the high energy expenditure during the pregnancy and lactation (8-13). Moreover, childbearing requires a lot of physical and mental resources, with detrimental effects on female health and longevity (29-33). Other studies have found no significant correlation between NOC and life span $(16,17)$. People in several countries or regions take contraception and birth control measures and do not give birth under the conditions of natural fertility, which might make the reproduction-longevity relationship complicated. As an island, Hainan has its unique historical, cultural, social, and economic characteristics: natural birth rates have been very high due to a lack of birth control, and the limited availability of medical resources has led to the limited use of medical interventions. It is worth noting that this presented a natural aging process. There were stable historical and cultural environment, and slow social and economic development. A time lag of at least 10 years between fertile age of women aged 80-99 years and centenarian women has a relatively smaller effect, which gives us an opportunity to explore the relationship between female fertility and life span. Therefore, this study tracked fertility status of women aged over 80 years in Hainan to explore the relationship between female fertility and life span. We found that low NOC had a significant association with longevity, and childbearing might be a harmful factor for longevity, in Chinese oldestold population.

As per antagonistic pleiotropy theory, undesirable mutations in later life could accumulate if they are beneficial to reproduction at younger age (1). According to disposable soma theory, selective maintenance of somatic cells to promote reproduction and accelerated aging (34). The evolutionary theories of aging have suggested that delaying reproductive time can prolong life span. Previous studies have identified that late fertility had positive effects on post-reproductive survival (35-40). This study indicated that ICA and LCA were significantly higher in centenarian women than in women aged 80-99 years. In late pregnancy, the mother benefits from sharing blood with the fetus, with the recovery of aging organs due to the physiological changes in late childbearing and breastfeeding.

It is generally believed that E2 and T levels are beginning to rise at puberty and reach to the top at the age of 30-40 years. As people get older, E2 and T levels continue to decline faster and faster. It is still unclear what role E2 and T play in oldest-old population. This study suggested that E2 levels had a positive association with age and longevity, and serum $\mathrm{T}$ levels had a negative association with age and longevity in Chinese oldest-old population. High levels of endogenous estrogen at menopause can prevent or delay the onset of aging, cardiovascular and other disease $(41,42)$. The net effect of late menopause is usually increased life span. Additionally, microarray deoxyribonucleic acid analyses of women aged over 45 years have shown that genes related to delayed ovarian aging were also involved in successful aging (43). Thus, late menopause generally indicates a long life span (44-46).

The current study had some limitations. Firstly, age at menopause was not taken into account in this study. It was very difficult for oldest-old individuals to recall age at menopause or obtain this information from their friends, relatives, offspring, or population survey/medical records. Secondly, this crosssectional study had no long-term follow-up to determine deceased women. We actually do not know if women aged 80 99 years will be centenarian women in a few years.

\section{CONCLUSION}

Based on the data from the CHCCS, this study demonstrated that the centenarians had crucial characteristics of less and delayed childbearing, indicating a negative relationship between female fertility and longevity, in Chinese oldest-old population. Meanwhile, serum E2 levels were positively associated and serum $\mathrm{T}$ levels were negatively associated with longevity. The less and late childbearing might be a significant factor of prolonged life span, and centenarian longevity 
and successful aging might be promoted by reducing and delaying female childbearing.

\section{DATA AVAILABILITY STATEMENT}

The raw data supporting the conclusions of this article will be made available by the authors, without undue reservation.

\section{ETHICS STATEMENT}

The studies involving human participants were reviewed and approved by the Ethics Committee of Hainan Hospital of Chinese People's Liberation Army General Hospital (Sanya, Hainan; Number: 301hn11201601). The patients/participants provided their written informed consent to participate in this study.

\section{AUTHOR CONTRIBUTIONS}

QiaoZ, SF, QianZ, JT, YZ, and YY contributed to the study design, conducted the data collection and analyses, and drafted the paper. All authors contributed to the article and approved the submitted version.

\section{REFERENCES}

1. Williams GC. Pleiotropy, natural selection, and the evolution of senescence. Evolution (1957) 11(4):398-411 doi: 10.1111/j.1558-5646.1957.tb02911.x.

2. Kirkwood TBL. Evolution of aging. Nature (1977) 270:233-8. doi: 10.1038/ $270301 \mathrm{a} 0$

3. Gordon EH, Peel NM, Chatfield MD, Lang IA, Hubbard RE. Frailty: A cost incurred by reproduction? Sci Rep (2020) 10(1):10139. doi: 10.1038/s41598020-67009-2

4. van den Heuvel J, English S, Uller T. Disposable soma theory and the evolution of maternal effects on ageing. PLoS One (2016) 11(1):e0145544. doi: 10.1371/journal.pone.0145544

5. Kaptijn R, Thomese F, Liefbroer AC, Van Poppel F, Van Bodegom D, Westendorp RG. The trade-off between female fertility and longevity during the epidemiological transition in the Netherlands. PLoS One (2015) 10(12):e0144353. doi: 10.1371/journal.pone.0144353

6. Thoppil H, Riabowol K. Senolytics: A translational bridge between bellular senescence and organismal aging. Front Cell Dev Biol (2020) 7:367. doi: $10.3389 /$ fcell.2019.00367

7. Zokaei N, Grogan J, Fallon SJ, Slavkova E, Hadida J, Manohar S, et al. Shortterm memory advantage for brief durations in human APOE $€ 4$ carriers. Sci Rep (2020) 10(1):9503. doi: 10.1038/s41598-020-66114-6

8. Helle S, Lummaa V, Jokela J. Are reproductive and somatic senescence coupled in humans? Late, but not early, reproduction correlated with longevity in historical Sami women. Proc Biol Sci (2005) 272(1558):29-37. doi: 10.1098/rspb.2004.2944

9. Hurt LS, Ronsmans C, Thomas SL. The effect of number of births on women's mortality: systematic review of the evidence for women who have completed their childbearing. Popul Stud (Camb) (2006) 60(1):55-71. doi: 10.1080/ 00324720500436011

10. Jasienska G. Reproduction and lifespan: Trade-offs, overall energy budgets, intergenerational costs, and costs neglected by research. Am J Hum Biol (2009) 21(4):524-32. doi: 10.1002/ajhb.20931

\section{FUNDING}

This work was supported by grants from the National Natural Science Foundation of China (81900357, 81903392, 81941021, 81901252, and 82001476), the Military Medical Science and Technology Youth Incubation Program (20QNPY110), the National Key R\&D Program of China (2018YFC2000400), the National S\&T Resource Sharing service platform Project of China (YCZYPT[2018]07), the General Hospital of PLA Medical Big Data R\&D Project (MBD2018030), the China Postdoctoral Science Foundation funded project (2019M650359), the National Geriatric Disease Clinical Medicine Research Center Project (NCRCG-PLAGH-2017-014), the Central Health Care Scientific Research Project (W2017BJ12), the Hainan Medical and Health Research Project (16A200057), the Sanya Medical and Health Science and Technology Innovation Project (2016YW21, 2017YW22, and 2018YW11), the Military Medicine Youth Program of Chinese PLA General Hospital (QNF19069), and the Clinical Scientific Research Supporting Fund of Chinese PLA General Hospital (2017FC-CXYY-3009). The sponsors had no role in the design, conduct, interpretation, review, approval, or control of this article.

\section{ACKNOWLEDGMENTS}

We appreciate all the staff of CHCCS for their continued cooperation and contributions in field work.

11. Gagnon A. Natural fertility and longevity. Fertil Steril (2015) 103(5):1109-16. doi: 10.1016/j.fertnstert.2015.03.030

12. Le Bourg E. Does reproduction decrease longevity in human beings? Ageing $\operatorname{Res} \operatorname{Rev}(2007)$ 6(2):141-9. doi: 10.1016/j.arr.2007.04.002

13. Alter G, Dribe M, Van Poppel F. Widowhood, family size, and post-reproductive mortality: a comparative analysis of three populations in nineteenth-century Europe. Demography (2007) 44(4):785-806. doi: 10.1353/dem.2007.0037

14. Westendorp RGJ, Kirkwood TBL. Human longevity at the cost of reproductive success. Nature (1998) 396:743-6. doi: 10.1038/25519

15. Gavrilov LA, Gavrilova NS. Is there a reproductive cost for human longevity? Anti Aging Med (1999) 2:121-3 doi: 10.1089/rej.1.1999.2.121.

16. Holt-Lunstad J, Smith TB, Layton JB. Social relationships and mortality risk: a meta-analytic review. PLoS Med (2010) 7(7):e1000316. doi: 10.1371/ journal.pmed.1000316

17. Ronsmans C, Khlat M, Kodio B, Ba M, De Bernis L, Etard J. Evidence for a 'healthy pregnant woman effect' in Niakhar, Senegal? Int J Epidemiol (2001) 30(3):467-73; discussion 474-465. doi: 10.1093/ije/30.3.467

18. Westendorp RGJ, Kirkwood TBL. Human longevity and reproductive success: response to Gavrilov and Gavrilova. Anti Aging Med (1999) 2:125-6. doi: 10.1089/rej.1.1999.2.125

19. Grundy E, Kravdal O. Reproductive history and mortality in late middle age among Norwegian men and women. Am J Epidemiol (2008) 167(3):271-9. doi: 10.1093/aje/kwm295

20. Evert J, Lawler E, Bogan H, Perls T. Morbidity profiles of centenarians: survivors, delayers, and escapers. J Gerontol A Biol Sci Med Sci (2003) 58:2327. doi: $10.1093 /$ gerona/58.3.M232

21. Ismail K, Nussbaum L, Sebastiani P, Andersen S, Perls T, Barzilai N, et al. Compression of morbidity is observed across cohorts with exceptional longevity. J Am Geriatr Soc (2016) 64:1583-91. doi: 10.1111/jgs.14222

22. Motta M, Bennati E, Ferlito L, Malaguarnera M, Motta L. Successful aging in centenarians:myths and reality. Arch Gerontol Geriatr (2005) 40:241-51. doi: 10.1016/j.archger.2004.09.002 
23. Jopp DS, Park MK, Lehrfeld J, Paggi ME. Physical, cognitive, social and mental health in near-centenarians and centenarians living in New York City: findings from the Fordham Centenarian Study. BMC Geriatr (2016) 16:1. doi: 10.1186/s12877-015-0167-0

24. Fu S, Yu H, Li Y, Lv F, Deng J, Zhang F, et al. Multiple measures of mineral metabolism were associated with renal function in Chinese centenarians: A cross-sectional study. Front Med (2020) 7:120. doi: 10.3389/fmed.2020.00120

25. Fu S, Yao Y, Lv F, Zhang F, Zhao Y, Luan F. Relationships of sex hormone levels with activity of daily living in chinese female centenarians. J Nutr Health Aging (2019) 23:753-7. doi: 10.1007/s12603-019-1247-1

26. Zhang C, Fu S, Zhao M, Liu D, Zhao Y, Yao Y. Associations between complement components and vitamin $\mathrm{D}$ and the physical activities of daily living Among a longevous population in Hainan, China. Front Immunol (2020) 11:1543. doi: 10.3389/fimmu.2020.01543

27. Fu S, Yao Y, Lv F, Zhang F, Zhao Y, Luan F. Serum homocysteine levels had important associations with activity and quality of daily living in Chinese centenarians. J Nutr Health Aging (2019) 23(5):479-82. doi: 10.1007/s12603019-1189-7

28. Mangel M. Complex adaptive systems, aging and longevity. J Theor Biol (2001) 213(4):559-71. doi: 10.1006/jtbi.2001.2431

29. Dribe M. Long-term effects of childbearing on mortality: evidence from preindustrial Sweden. Popul Stud (Camb) (2004) 58(3):297-310. doi: 10.1080/ 0032472042000272357

30. Jasienska G, Nenko I, Jasienski M. Daughters increase longevity of fathers, but daughters and sons equally reduce longevity of mothers. Am J Hum Biol (2006) 18(3):422-5. doi: 10.1002/ajhb.20497

31. Korpelainen H. Human life histories and the demographic transition: a case study from Finland, 1870-1949. Am J Phys Anthropol (2003) 120(4):384-90. doi: 10.1002/ajpa.10191

32. Drenos F, Westendorp RG, Kirkwood TB. Trade-off mediated effects on the genetics of human survival caused by increasingly benign living conditions. Biogerontology (2006) 7(4):287-95. doi: 10.1007/s10522-006-9027-9

33. Gavrilova NS, Gavrilov LA. Human fertility and reproduction an evolutionary perspective. In: E Voland, A Chasiotis, W Schiefenhoevel, editors. Grandmotherhood: the evolutionary significance of the second half female life. New Brunswick: Rutgers University Press (2005). p. 59-80.

34. Kirkwood TB. Evolution of ageing. Nature (1977) 270:301-4. doi: 10.1038/270301a0

35. Smith KR, Mineau GP, Bean LL. Fertility and post-reproductive longevity. Soc Biol (2002) 49(3-4):185-205. doi: 10.1080/19485565.2002.9989058

36. Perls TT, Alpert L, Fretts RC. Middle-aged mothers live longer. Nature (1997) 389(6647):133. doi: 10.1038/38148

37. Muller HG, Chiou JM, Carey JR, Wang JL. Fertility and life span: late children enhance female longevity. J Gerontol A Biol Sci Med Sci (2002) 57(5):B202206. doi: 10.1093/gerona/57.5.B202
38. McArdle PF, Pollin TI, O’Connell JR, Sorkin JD, Agarwala R, Schaffer AA, et al. Does having children extend life span? A genealogical study of parity and longevity in the Amish. J Gerontol A Biol Sci Med Sci (2006) 61(2):190-5. doi: 10.1093/gerona/61.2.190

39. Gogele M, Pattaro C, Fuchsberger C, Minelli C, Pramstaller PP, Wjst M. Heritability analysis of life span in a semi-isolated population followed across four centuries reveals the presence of pleiotropy between life span and reproduction. J Gerontol A Biol Sci Med Sci (2011) 66(1):26-37. doi: 10.1093/gerona/glq163

40. Jaffe D, Kogan L, Manor O, Gielchinsky Y, Dior U, Laufer N. Influence of lateage births on maternal longevity. Ann Epidemiol (2015) 25(6):387-91. doi: 10.1016/j.annepidem.2014.12.002

41. Gielchinsky Y, Laufer N, Weitman E, Abramovitch R, Granot Z, Bergman Y, et al. Pregnancy restores the regenerative capacity of the aged liver via activation of an mTORC1-controlled hyperplasia/hypertrophy switch. Genes Dev (2010) 24(6):543-8. doi: 10.1101/gad.563110

42. Saltiki K, Alevizaki M. Coronary heart disease in postmenopausal women; the role of endogenous estrogens and their receptors. Hormones (Athens) (2007) 6 (1):9-24.

43. Gielchinsky Y, Bogoch Y, Rechavi G, Jacob-Hirsch J, Amariglio N, Shveiky D, et al. Gene expression in women conceiving spontaneously over the age of 45 years. Fertil Steril (2008) 89(6):1641-50. doi: 10.1016/ j.fertnstert.2007.06.058

44. Perls TT, Fretts RC. The evolution of menopause and human life span. Ann Hum Biol (2001) 28(3):237-45. doi: 10.1080/030144601300119052

45. Ossewaarde ME, Bots ML, Verbeek AL, Peeters PH, van der Graaf Y, Grobbee $\mathrm{DE}$, et al. Age at menopause, cause-specific mortality and total life expectancy. Epidemiology (2005) 16(4):556-62. doi: 10.1097/01.ede.0000165392.35273.d4

46. Wu X, Cai H, Kallianpur A, Gao YT, Yang G, Chow WH, et al. Age at menarche and natural menopause and number of reproductive years in association with mortality: results from a median follow-up of 11.2 years among 31,955 naturally menopausal Chinese women. PLoS One (2014) 9(8): e103673. doi: 10.1371/journal.pone.0103673

Conflict of Interest: The authors declare that the research was conducted in the absence of any commercial or financial relationships that could be construed as a potential conflict of interest.

Copyright (c) 2021 Zhu, Fu, Zhang, Tian, Zhao and Yao. This is an open-access article distributed under the terms of the Creative Commons Attribution License (CC BY). The use, distribution or reproduction in other forums is permitted, provided the original author(s) and the copyright owner(s) are credited and that the original publication in this journal is cited, in accordance with accepted academic practice. No use, distribution or reproduction is permitted which does not comply with these terms. 